\title{
A Case of Painful Post-Traumatic Trigeminal Neuropathy after Endoscopic Sinus Surgery
}

\author{
Junho Hwang, ${ }^{1}$ Taehun Lim ${ }^{1}$, Joon Bum Joo ${ }^{1}$, and Woo Hyun Lee ${ }^{2} \mathbb{D}$ \\ ${ }^{1}$ Department of Otolaryngology, National Police Hospital, Seoul; and ${ }^{2}$ Department of Otolaryngology, \\ Kangwon National University Hospital, Chuncheon, Korea \\ 부비동 내시경 수술 후 발생한 통증성 외상후 삼차신경병증 1예 \\ 황준호 ${ }^{1} \cdot$ 임태헌 $^{1} \cdot$ 주준범 ${ }^{1} \cdot$ 이우현 ${ }^{2}$ \\ 국립경찰병원 이비인후과, ${ }^{1}$ 강원대학교병원 이비인후과 ${ }^{2}$
}

\author{
Received April 6, 2020 \\ Revised June 3, 2020 \\ Accepted June 11, 2020 \\ Address for correspondence \\ Woo Hyun Lee, MD, PhD \\ Department of Otolaryngology, \\ Kangwon National \\ University Hospital, \\ 156 Baengnyeong-ro, \\ Chuncheon 24289, Korea \\ Tel +82-33-258-9109 \\ Fax +82-33-258-9486 \\ E-mail sniper212@hanmail.net
}

Painful post-traumatic trigeminal neuropathy (PPTTN) is a distinctive facial pain syndrome characterized by facial and/or oral pain along the sensory distribution of the fifth cranial nerve with a clear history of a traumatic event. To our knowledge, PPTTN after endoscopic sinus surgery (ESS) has not been reported in the literature. We present a case of an elderly woman who experienced unilateral neuralgic pain after undergoing ESS. Following ESS of the left sphenoid sinus to remove suspicious fungus balls, the patient complained of intermittent facial pain occurring in the left periorbital and forehead area. Clinical diagnosis of PPTTN was established after a comprehensive multidisciplinary assessment. The pain disappeared completely after a 5 -week medical treatment with gabapentin. Since PPTTN cases associated with ESS are extremely rare, our case demonstrates that PPTTN should be considered in the differential diagnosis of facial pain developing after ESS and can be successfully treated with medical therapy. Korean J Otorhinolaryngol-Head Neck Surg 2021;64(4):268-72

Key Words Facial pain · Mycoses · Paranasal sinus diseases · Sphenoid sinusitis Trigeminal nerve diseases.

\section{Introduction}

Painful post-traumatic trigeminal neuropathy (PPTTN) is a clinical pain syndrome that is characterized by a localized facial and/or oral pain along the distribution of trigeminal nerve with a history of an identifiable traumatic event. The pain may have burning or shooting-like qualities, accompanied by positive (hyperalgesia, allodynia) and/or negative (hypaesthesia, hypalgesia) changes in the neurologic profile, and its duration ranges widely from paroxysmal to constant. The incidence of painful neuropathy following injuries to the peripheral branch-

This is an Open Access article distributed under the terms of the Creative Commons Attribution Non-Commercial License (https://creativecommons.org/licenses/by-nc/4.0) which permits unrestricted non-commercial use, distribution, and reproduction in any medium, provided the original work is properly cited. es of the trigeminal nerve is reported at around $3-5 \%$. $^{1-5)}$

The etiology of PPTTN varies widely according to the cause of injury. In a previous study with 91 PPTTN cases, 67 occurred after dental procedures (including 4 cases of maxillary sinus surgeries), and 24 developed by major traumas such as traffic accidents or assaults. ${ }^{3)}$ In a more recent study with 97 PPTTN patients, the majority of cases occurred after dental interventions (including third molar surgery, tooth extraction, implant placement, endodontic treatment), and only 2 cases occurred after ear/nasal surgeries." ${ }^{4)}$ Although rare, PPTTN has been more commonly reported in the field of dentistry, since peripheral branches of the trigeminal nerve (e.g., the inferior alveolar nerve or lingual nerve) can readily be damaged during invasive dental procedures. ${ }^{3,6)}$ 
We present a case of a woman who experienced PPTTN after endoscopic sinus surgery (ESS). There have been a few case reports of neuralgic pain or numbness along the distribution of the trigeminal nerve associated with paranasal sinusitis; ${ }^{7,8)}$ however, to our knowledge, PPTTN after ESS of the sphenoid sinus has not been reported in the literature. The clinical significance of this case report lies not only in its rarity, but also in sharing the knowledge that this less known disease can present with severe manifestations and poor prognosis.

\section{Case}

A 61-year-old female patient visited our clinic with postnasal drip that persisted for 1 year. The patient did not have headache or any neuralgic pain. Aside from taking antidepressants due to depression, she had no significant medical, surgical, familial, or social history. Endoscopic examination of the nasal cavity showed mucopurulent discharge in the left nasal cavity and nasal septal deviation to the left side. Ostiomeatal unit (OMU) CT revealed calcifications in the left sphenoid sinus and a small bony defect in the lateral wall of the left sphenoid sinus (Fig. 1). Skin prick tests were performed to diagnose allergic rhinitis, but no offending allergen was detected. Left sphenoidotomy was performed via the transostial approach under general anesthesia. When the left sphenoid sinus ostium was widened, dark brown-colored fungal debris were observed and completely removed. Mucosal lining of the sinus was intact and mild swelling was observed. No postoperative nasal packing was used. The patient was discharged the day after ESS.

The patient visited the emergency department on the fifth

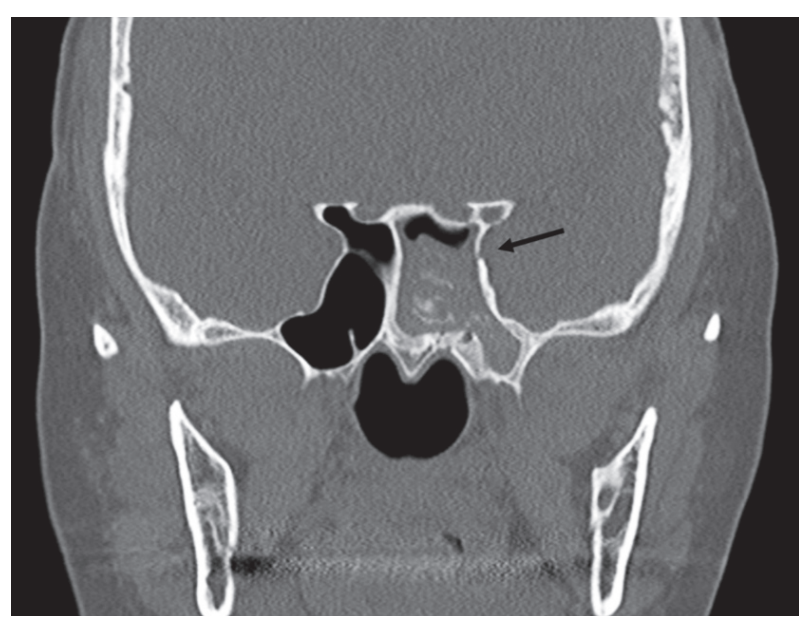

Fig. 1. Findings of preoperative ostiomeatal unit CT. Calcifications in the left sphenoid sinus and a small bony dehiscence of the lateral sphenoid sinus wall are seen (arrow). postoperative day with squeezing facial pain localized in the left periorbital and forehead area, consistent with the innervation territory of the ophthalmic branch (V1) of the trigeminal nerve. The pain occurred 5 to 6 times a day, usually lasted for less than 30 minutes, and showed 5- to 6-points on the Numerical Rating Scale (NRS) where 0 indicates no pain and 10 the worst pain. Softly brushing a cotton-tipped swab against the affected area elicited a pain response. Facial palsy was not observed. Endoscopic examination revealed patent sphenoid ostium. Follow-up OMU CT revealed no calcifications in the left sphenoid sinus, suggesting complete eradication of the fungus balls (Fig. 2). She was readmitted with a clinical suspicion of herpes zoster ophthalmicus, due to neuropathic pain affecting the V1 dermatome. Valaciclovir (1000 mg tid) and gabapentin (100 mg tid) were administered.

Multidisciplinary evaluation was attempted after initial treatment. An infectious disease specialist concluded that the clinical features do not correlate with herpes zoster ophthalmicus, since the patient showed no viral prodrome, periocular cutaneous rash, or any abnormal findings on a complete ophthalmic examination. On account of the typical characteristics of the pain (i.e., pain development immediately after an identifiable traumatic event, a clinically evident positive sign of trigeminal nerve dysfunction, and the pain distribution consistent with the innervation territory of the nerve), a neurologist suggested the possibility of PPTTN in the ophthalmic division. Blink reflex test and orbital MRI were performed to rule out the possibility of secondary trigeminal neuralgia (TN). Latencies of trigeminal reflex were within normal limits $(\mathrm{R} 1=$ $11.3 \mathrm{~ms}, \mathrm{R} 2=36.0 \mathrm{~ms}$; normal range 9.1-12.4 ms and 28.0-

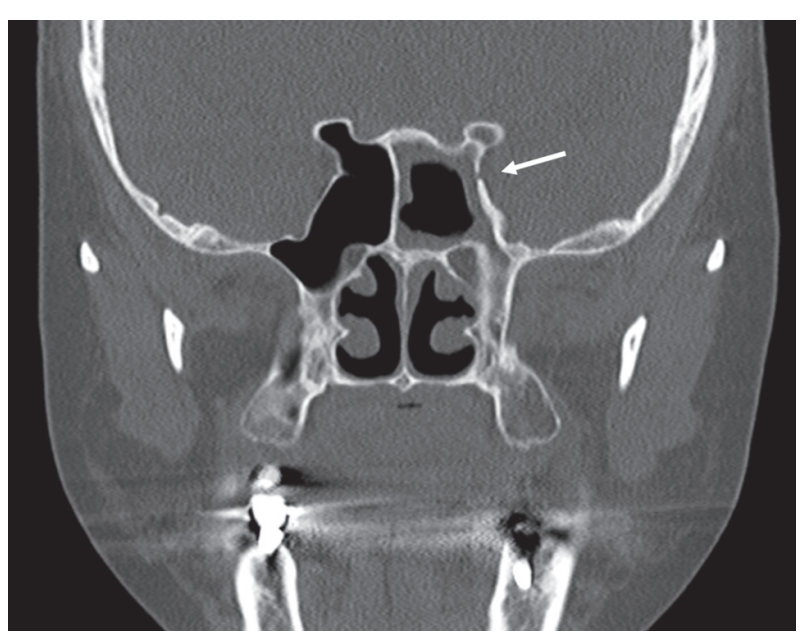

Fig. 2. Findings of postoperative ostiomeatal unit CT. Disappearance of calcifications in left sphenoid sinus is seen, but the lateral wall bony dehiscence (arrow) and mucosal thickenings (probably postoperative change) still exist. 
$41.0 \mathrm{~ms}$, respectively), and the trigeminal nerve was intact on the MRI (Fig. 3). Since using antiepileptic drugs is one of the mainstays of PPTTN treatment, gabapentin dosage was increased threefold (300 mg tid) and zaltoprofen (80 mg tid) was added.

The pain subsided to a tolerable level (NRS 1-2 points at each episode) after 8 days of treatment, and the patient was discharged from the hospital. The patient continued to take the medications with decreased dosage (gabapentin $100 \mathrm{mg}$ tid and zaltoprofen $80 \mathrm{mg}$ tid) for 4 weeks after discharge. The pain disappeared completely in the sixth postoperative week. There was no evidence of recurrence during 12-months of follow-up.

This case report was performed under the Kangwon National University Hospital Ethics Board guidelines for case reports. No formal research ethics board approval was necessary and therefore no reference number was generated. This study has been conducted ethically according to the principles of the Declaration of Helsinki.

\section{Discussion}

Until recently, the term PPTTN has tended to be used somewhat interchangeably with $\mathrm{TN}$ due to similarities in their pain characteristics. However, according to the latest International Classification of Headache Disorders (ICHD-3), the two entities differs in duration (TN: lasts from a fraction of a second to two minutes; PPTTN: ranges widely from paroxysmal to constant, and may be mixed), associated nerve dysfunction
(TN: rare; PPTTN: positive and/or negative changes) and pain quality (TN: electric-shock like, stabbing or shooting; PPTTN: burning, squeezing or "needles and pins"). MRI or electrophysiological test can be useful for detecting underlying causes of TN (e.g., neurovascular compression with morphological changes in the trigeminal nerve root on MRI in classical TN, plaque on MRI or longer latencies on the blink reflex test in secondary TN attributed to multiple sclerosis, etc.). ${ }^{1,49)}$ In our case, typical pain characteristics were sufficient to clinically diagnose PPTTN, and the tests performed to further exclude possible etiologies of TN revealed no notable abnormalities.

Herpes zoster ophthalmicus can also be considered in patients with neuropathic pain (e.g., burning, shooting, or tingling pain) that is limited to V1 dermatome. Periocular cutaneous rash is an important hallmark of the disease. The rash commonly appears over the eyelids, medial canthal area, and most importantly, the tip of the nose, which highly suggests ophthalmic involvement (Hutchinson sign). A thorough ophthalmic evaluation is crucial, since the cutaneous reaction can affect cornea or retina and can lead to permanent loss of vision. Viral prodrome such as fever, malaise, or fatigue may precede cutaneous lesions or neuropathic pain. ${ }^{10)}$ Our patient showed no viral prodrome, periocular rash, or any abnormalities on ophthalmic examination.

The pathophysiology of PPTTN is not fully elucidated. A cascade of functional, biochemical, physical and genetic alterations in peripheral neurons is suggested to be involved and may result in central changes. Inflammatory molecules following nerve injury can result in reversible sensitization of

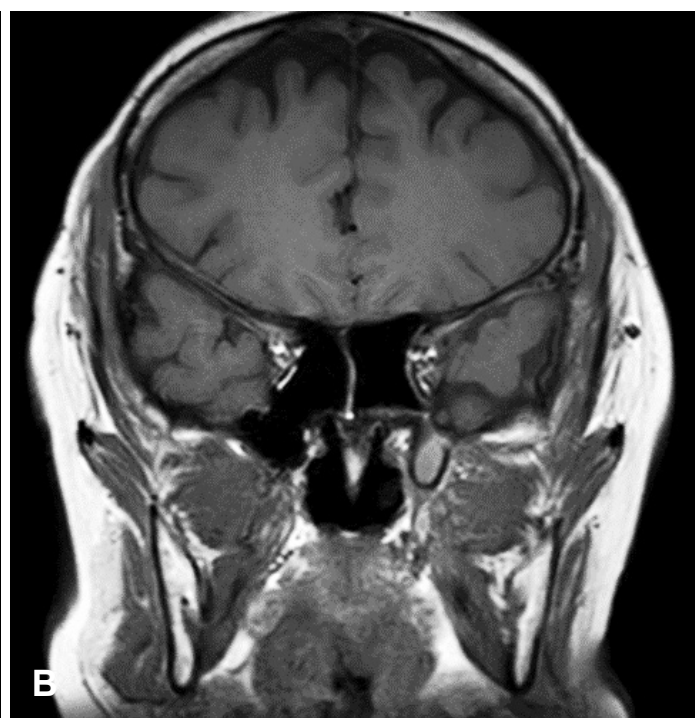

Fig. 3. Findings of postoperative orbital MRI. There are no significant abnormal findings in the optic nerves, Meckel's caves, and cavernous sinus on T2-weighted axial view (A) and T1-weighted coronal view (B). The trigeminal nerves were intact (arrows) and no morphological changes were identified along the anatomical course of the nerves. 
nociceptors (peripheral sensitization). Examples of peripheral sensitization include hyperalgesia and allodynia. Disorganized healing of damaged nerve can also induce neuroma formation and focal demyelination, and those regions can generate ectopic impulses, resulting in spontaneous neuropathic pain. Neuropeptide expression alteration that can induce functional modifications may occur following nerve injury (e.g., allodynia as a result of substance P expression in A $\beta$ fibers). Repeated primary nociceptive afferent input could eventually sensitize the central nervous system, resulting in hypersensitive responses (central sensitization). ${ }^{11)}$

In a rare case of severe neuralgic pain and decreased sensation in the distribution of the trigeminal nerve associated with severe acute sphenoid sinusitis, there is a possibility that severe inflammation in the sphenoid sinus in close proximity to the area of the trigeminal nerve could affect the nerve. ${ }^{7)}$ In another case of numbness along the second branch of the trigeminal nerve associated with acute sphenoid sinusitis, a bony wall separating the affected sphenoid sinus from the adjacent foramen rotundum was unclear, and possibly inflammation in the sphenoid sinus involved the trigeminal nerve through the foramen. ${ }^{8)}$ In our case, a preoperative bony dehiscence at the lateral wall of the left sphenoid sinus could have served as postoperative inflammation route. Otherwise, ESS itself or intraoperative saline irrigation could have damaged the trigeminal nerve either directly or indirectly by applying pressure. It is also possible that postoperative swelling could have resulted in pressure-induced nerve damage.

Medical treatment of PPTTN primarily consists of antiepileptic drugs, tricyclic antidepressants and newer antidepressants. If the patient's previous medical history precludes the use of an antidepressant, anticonvulsants such as gabapentin and pregabalin are recommended. Since the patient was on antidepressants, we used gabapentin to control the pain and it effectively alleviated neuralgia on the left V1 area. The role of surgical treatment for painful trigeminal neuropathies remains unclear and needs to be further investigated. Psychotherapeutic support is recommended to distressed patients, despite the evidence is still lacking., ${ }^{2,5,11)}$

The prognosis of PPTTN is unfavorable. According to a prospective cohort study, response rate of PPTTN to current pharmacotherapy was limited (significant pain relief in $11 \%$ of the patients) and inferior to that of classical TN. ${ }^{12)}$ However, our patient experienced a complete remission of pain, hinting at the possibility that minor nerve damage without direct injury can be successfully managed with medical treatment.
One of the limitations of this study includes the inherent uncertainty of clinical diagnosis. With no definitive diagnostic tests, the diagnosis relies heavily on the clinician's impression. Also, due to the rarity of this case, it is hard to establish a definite link of causality. Since the natural history of PPTTN is not fully elucidated and, to the best of our knowledge, PPTTN after ESS is first to be reported, it is not certain whether the patient underwent spontaneous remission of the disease, or whether the disease responded to the treatment with gabapentin. Therefore, more cases should be accumulated to better understand the nature of the disease and to determine the most appropriate treatment strategy.

Our case demonstrated that reversible damage to the trigeminal nerve may occur after ESS and can be successfully managed with medical treatment. Although rare, PPTTN should be considered in the differential diagnosis in patients presenting with facial pain after ESS. Thorough preoperative and postoperative imaging studies may be helpful in recognizing putative etiologies of PPTTN such as a dehiscence of the sphenoid sinus wall adjacent to the trigeminal nerve. Medical treatment can be attempted with anticonvulsants and antidepressants.

\section{Acknowledgments}

We would like to express our deepest gratitude to Dr. Yang-Gi Min for correcting errors in this manuscript.

\section{Author Contribution}

Conceptualization: all authors. Data curation: Junho Hwang, Taehun Lim, Woo Hyun Lee. Formal analysis: Junho Hwang, Joon Bum Joo, Woo Hyun Lee. Funding acquisition: Joon Bum Joo, Woo Hyun Lee. Investigation: Junho Hwang, Taehun Lim, Woo Hyun Lee. Methodology: Woo Hyun Lee. Project administration: Woo Hyun Lee. Resources: Joon Bum Joo, Woo Hyun Lee. Software: Taehun Lim, Woo Hyun Lee. Supervision: Joon Bum Joo, Woo Hyun Lee. Validation: Joon Bum Joo, Woo Hyun Lee. Visualization: Junho Hwang, Taehun Lim, Woo Hyun Lee. Writing - original draft: Junho Hwang, Woo Hyun Lee. Writing — review \& editing: Junho Hwang, Woo Hyun Lee.

\section{ORCID}

Woo Hyun Lee

https://orcid.org/0000-0001-7725-4146

\section{REFERENCES}

1) Headache Classification Committee of the International Headache Society (IHS) The International Classification of Headache Disorders, 3rd edition. Cephalalgia 2018;38(1):1-211.

2) International Association for the Study of Pain (IASP). Painful post-Traumatic Trigeminal Neuropathy (PTTN). Orofacial Pain Fact Sheets [online] 2016 Sep [cited 2020 Jun 3]. Available from: URL: https://s3.amazonaws.com/rdcms-iasp/files/production/ public/Content/ContentFolders/GlobalYearAgainstPain2/20132014 OrofacialPain/FactSheets/PTTN_2016.pdf.

3) Benoliel R, Zadik Y, Eliav E, Sharav Y. Peripheral painful traumatic trigeminal neuropathy: Clinical features in 91 cases and proposal of 
novel diagnostic criteria. J Orofac Pain 2012;26(1):49-58.

4) Melek LN, Smith JG, Karamat A, Renton T. Comparison of the neuropathic pain symptoms and psychosocial impacts of trigeminal neuralgia and painful posttraumatic trigeminal neuropathy. J Oral Facial Pain Headache 2019;33(1):77-88.

5) Benoliel R, Kahn J, Eliav E. Peripheral painful traumatic trigeminal neuropathies. Oral Dis 2012;18(4):317-32.

6) Pogrel MA, Jergensen R, Burgon E, Hulme D. Long-term outcome of trigeminal nerve injuries related to dental treatment. J Oral Maxillofac Surg 2011;69(9):2284-8.

7) Sawaya RA. Trigeminal neuralgia associated with sinusitis. ORL J Otorhinolaryngol Relat Spec 2000;62(3):160-3.
8) Hirose Y, Hirota Y, Yokokawa D, Ohira Y, Ikusaka M. Acute sphenoiditis involving the second branch of the trigeminal nerve. QJM 2016;109(11):745-6.

9) Cruccu G. Trigeminal neuralgia. Continuum (Minneap Minn) 2017; 23(2, Selected Topics in Outpatient Neurology):396-420.

10) Vrcek I, Choudhury E, Durairaj V. Herpes zoster ophthalmicus: A review for the internist. Am J Med 2017;130(1):21-6.

11) Benoliel R, Teich $S$, Eliav E. Painful traumatic trigeminal neuropathy. Oral Maxillofac Surg Clin North Am 2016;28(3):371-80.

12) Haviv Y, Zadik Y, Sharav Y, Benoliel R. Painful traumatic trigeminal neuropathy: An open study on the pharmacotherapeutic response to stepped treatment. J Oral Facial Pain Headache 2014;28(1):52-60. 